\title{
Le jeu des pouvoirs ou pourquoi Hérodias
}

Magalie D. Hanquier

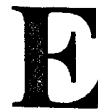

n lisant Hérodias, dernier des Trois Contes de Flaubert, les critiques se sont souvent interrogés sur la hiérarchie des personnages. Certes, Hérodias, l'épouse si peu légitime d'Antipas a donné son nom au texte, mais pour C.H. Wake par exemple, Antipas est en fait le personnage central (322). ${ }^{1,2}$ En revanche, selon Jane Robertson, Antipas représente "l'homme moyen" et c'est la tension entre Hérodias, femme prise dans la temporalité et le monde de l'Ancien Testament, Jézabel et Iaokanann, prophète si peu humain d'un nouveau monde, celui du nouveau testament, qui sous-tend la structure du conte..$^{3}$

Cette difficulté que nous avons de découvrir un protagoniste ne tientelle pas au fait que les rôles de chaque personnage évoluent au cours du récit? De leurs actes, de leurs désirs et de leurs rapports ne naît-il pas un renversement de hiérarchie qui modifie leur importance dans l'économie du texte?

Peut-être en étudiant le jeu des pouvoirs dans Hérodias et ses multiples redistributions, tant au niveau politique que religieux, parviendrons-nous à élucider le mystère du titre. A travers l'éclairage que nous pourrons donner sur l'organisation hiérarchique des personnages nous tenterons de découvrir un sens nouveau à l'utilisation de cet épisode biblique par Flaubert.

La vision qui débute Hérodias a été longuement commentée par les critiques car de nombreux éléments du récit y sont rassemblés. On sait que Flaubert déclara la voir véritablement. ${ }^{4} L e$ pouvoir d'Antipas apparaît d'abord immense, il domine du haut de son nid d'aigle les provinces soumises à sa juridiction, inaltérable sur son bloc de basalte comme un seigneur du Moyen-Age dans son château-fort entouré de "[q]uatre vallées profondes" (139) qui tiennent lieu de fossés. Même la balustrade de sa terrasse est en bois de sycomore réputé incorruptible. Le soleil en se levant "derrière Machaerous" (140) lui fait comme une couronne flamboyante. Ses provinces semblent remplies comme une come d'abondance.

Mais déjà la première scène nous annonce combien le pouvoir d'Antipas est menacé. Machaerous est "suspendue au-dessus de l'abîme" (139) 
et ce mot est repris plusieurs fois: "leur masse jusqu'au fond des abîmes, était encore dans l'ombre" (140), "la profondeur des abîmes le troublaient" (145). La Tour Antonia, symbole de la puissance des Romains, lui rappelle qu'il tient son pouvoir de ces derniers. Quant au temple de Jérusalem, il évoque ces Juifs qui "ne voulaient plus de ses moeurs idolâtres" (142). Surtout, au-delà des menaces tangibles que ces peuples représentent, un angoissant mystère semble émaner des profondeurs de l'ombre. Quand le brouillard se déchire, ce ne sont que "les contours de la Mer Morte" (140) qui apparaissent, mais "ensevelies plus bas que le rivage sous les eaux pesantes" (146) se trouvent les "villes maudites" (146). Si les "crêtes" (140) et les "surfaces raboteuses" (140) des montagnes expriment l'agressivité de ce pays aride, elles sont loin de provoquer autant l'inquiétude que "leur masse" (140) "encore dans l'ombre" (140).

Plus encore que des forces politiques hostiles ou dominantes Antipas peut donc craindre des forces occultes qui se sont jadis exprimées et menacent encore depuis les profondeurs de la terre. A ce point du récit, Antipas ne semble pas établir une claire hiérarchie des dangers qui l'accablent. En distingue-t-il même la nature différente?

La première menace sérieuse qu'il aperçoit de son balcon est incarnée dans "les troupes du roi de Arabes, dont il avait répudié la fille pour prendre Hérodias" (141). Cette dernière constitue donc la cause du conflit. En fait, depuis son arrivée, Hérodias semble avoir érodé peu à peu la puissance d'Hérode par sa simple présence. De leurs amours "tous ses malheurs ..... découlaient" (148). En effet, Hérode est vieilli, voûté, tout en lui semble respirer la tristesse, sa "toge sombre" (148), son "front chagrin" (148-49). Il paraît sans force, sans projet, vide et sans avenir.

Pourtant, Hérodias se présente comme un allié politique enthousiaste qui ne prend pas le temps de se parer pour lui annoncer avec joie l'emprisonnement de son propre frère, Agrippa, et par là même la fin de ses craintes vis-àvis de l'Empereur. Elle a fait plus: "les clients achetés, les lettres découvertes, des espions à toutes les portes, et comment elle était parvenue à séduire Eutychès le dénonciateur" (147). Pour unir son sort à celui d'Hérode, elle a même abandonné sa propre fille dans Rome. Hérodias prétend donc contribuer au pouvoir d'Antipas et cela paraît d'ailleurs être son intérêt. Mais Hérode se méfie. S'il justifie politiquement les actions d'Hérodias, il ne s'enthousiasme pas de son aisance à les accomplir. Il ne semble pas accorder beaucoup de crédit à sa soudaine tendresse maternelle. Surtout, "il la repoussa" (148), nous dit le narrateur. Antipas semble éprouver plus de dégoût que de lassitude pour elle. Nous sentons donc dès les premières pages du conte que le duo politique HérodeHérodias comporte des failles qui pourraient avoir des conséquences sur leur possession du pouvoir. En fait, on peut dire qu'Hérodias mine le pouvoir d'Antipas: indirectement et extérieurement en provoquant la guerre (sorte d'Hélène) et directement en ne suscitant plus que de la répulsion chez Antipas, source permanente de tristesse à l'intérieur du palais.

L'opposition d'Antipas et d'Hérodias apparaît clairement dans l'enchaînement de l'ordre et du contre-ordre adressés à Mannaëi: "Tue-le" - "Arrête" (149). Dès lors, Iaokanann apparaît comme l'objet sur lequel se cristallise leur opposition. Mais c'est encore à Antipas que Mannaëi obéit. Or Mannaëi est le bourreau, la main par laquelle s'exerce le droit de vie et de mort du souverain sur 
ses sujets. Flaubert nous l'a déjà longuement décrit: "ses dents brillaient, et ses orteils posaient légèrement sur les dalles, tout son corps ayant la souplesse d'un singe, et sa figure l'impassibilité d'une momie. (143). Il me semble qu'il est aussi l'archétype du soldat antique tel que Flaubert l'imaginait déjà dans Salammbô, rusé, félin et d'une sauvagerie carnassière. Il est donc bien la main armée du pouvoir, celui sans lequel la puissance demeure sans effet, désarmée. Cependant, il est "vieux, décharné" (142), une "somnolence décolor[e] ses yeux" (143) et l'on pense aussitôt qu'Antipas se repose sur une arme fidèle mais usée. Hérodias, par ses récriminations contre l'indulgence d'Antipas envers Iaokanann, entraîne le Tétrarque dans une sordide querelle de ménage où il perd toute dignité: "J'ai pris un bon soutien, en entrant dans ta famille! " - "Elle vaut la tienne! " (152). Il est méprisé dans sa propre maison par son épouse, "exhalant le mépris de la patricienne pour le plébéien" (152): comment pourrait-on encore croire à son pouvoir?

Après qu'Antipas ait aperçu pour la première fois Salomé, un second indice nous est donné de l'attitude ambiguë d'Hérodias à son égard. Elle qui s'est vue repoussée par le Tétrarque, loin de s'alarmer des "flammes [qui] s'allumaient dans ses yeux" (154) ne semble ressentir aucune jalousie "et s'en [va] soudainement apaisée" (154). Or Hérode ne semble pas s'apercevoir de cette troublante réaction. Lui, qui pourtant se méfiait d'Hérodias au début de leur entretien, présente ici le même aveuglement qu'il montrera lors du banquet final. Bien qu'Antipas semble déjà conscient d'avoir commis une erreur en s'abandonnant à sa passion pour Hérodias, sa vigilance lui fait défaut dès que ses sens s'enflamment. L'on sent dès ce moment qu'Hérode met délibérément en danger son pouvoir politique parce qu'il manque de pouvoir sur lui-même. Comme le dit C. H. Wake, "a new dream emerges and suddenly takes possession of him" et "his will [is] not in control." $s$

Lorsqu'au chapitre II Vitellius apparait, Hérodias compromet les relations du souverain avec Rome. Elle "s'avançait d'un air d'impératrice" (159) nous dit le narrateur. Or, ceci ne peut que déplaire au Romain pour lequel il s'agit de demeurer "fidèle toujours aux Césars" (159). Son attitude hautaine ne demeure pas son unique maladresse, elle dévoile au Proconsul qu'elle en sait plus que lui sur les affaires de l'Etat, soulevant ainsi sa méfiance ("elle lui parut dangereuse" (159)) et ranimant son animosité envers Antipas. En se présentant comme une rivale de Rome, elle met en danger par son orgueil le pouvoir délégué à Hérode par César.

Seules les invectives de Iaokanann lient le couple royal. Ils subissent ensemble l'accusation d'adultère et d'inceste, d'autant moins contestable que le prophète peut ajouter: "[l]e châtiment est déjà dans ton inceste. Dieu t'afflige de la stérilité du mulet! " (174). Une fois encore, Hérodias qui "vaincue par une fascination" (171) s'était précipitée vers la fosse, couvre Antipas de ridicule car "[d]es rires s'élevèrent" (174). Notons qu'elle contrôle aussi peu son orgueil qu'Hérode sa sensualité. Pour évoquer la honte d'Antipas, Flaubert écrit: "Antipas paraissait mourir" (176). Mais il s'agit de bien plus qu'un cliché: le Tétrarque Antipas a réellement commencé son agonie.

Sa peur, sa honte et son sentiment d'impuissance l'ont déjà rendu manipulable par Hérodias dès le chapitre II. En effet, pour la plupart des critiques Antipas admire et respecte Iaokanann jusqu'à la danse de Salomé où il se trouve contraint de le condamner par sa promesse insensée. Mais en fait il ne me paraît plus se soucier de son sort lorsqu'il confirme l'affirmation mensongère 
d'Hérodias: "Il [Iaokanann] ordonne au peuple de refuser l'impôt!" (177). D'ailleurs, bien qu'Antipas dise de Iaokanann dans le chapitre I: "Sa puissance est forte!.... Malgré moi, je l'aime!" (156), il s'est déjà résolu à le combattre ("Puisqu'il m'attaque, je me défends" - 155) et affirme à son propos: "On ne relâche pas les bêtes furieuses!".(156). Dans cette perspective, la danse de Salomé pourrait ne constituer qu'un coup de grâce. Cependant, plus Hérodias constitue une nuisance politique pour Antipas, plus il parait s'en rapprocher. Afin de comprendre la logique d'Hérode, il devient nécessaire d'étudier leurs relations selon une autre perspective.

L'amour n'unit plus le Tétrarque à Hérodias, mais lorsqu'au chapitre II il recourt à elle pour lui donner "du courage" (180), l'on nous dit: "tous les liens n'étaient pas rompus de l'ensorcellement qu'il avait autrefois subi” (180). Quoique Hérode prétende haïr Hérodias, il ne peut s'en passer. Bien qu'elle ait perdu les attraits de sa jeunesse, qu'elle le méprise et qu'elle nuise à son gouvernement, elle lui demeure précieuse. En fait, bien qu'Antipas soit préfet de Rome, il n'appartient pas au monde rationnel de Vitellius. C'est un homme de l'Ancien Orient qui croit aux signes interprétés par les sages et pour lequel la réalité objective n'est pas la seule réalité. Aussi ne fait-il pas reposer son pouvoir uniquement sur des choix politiques mais essentiellement sur la protection de puissances mystérieuses. Lorsqu'il fait appel à Hérodias pour le rassurer et lui donner du courage, ce n'est donc pas, en l'occurence, à l'amante qu'il s'adresse mais à la sorcière. Tout comme il ne doute pas de la science de Phanuel, il est certain qu'Hérodias peut l'aider. Or, elle détient effectivement le talisman qui doit protéger sa vie et son pouvoir: "une médaille bizarre, ornée du profil de Tibère" (180). Quand le banquet tourne au pugilat, Antipas ne fera pas appel à l'armée, en souverain moderne, mais à la magicienne en brandissant l'amulette. Aussitôt, Hérodias apparaît et la fureur s'apaise ou plutôt se pétrifie. Le geste d'Antipas était incantatoire et la parole d'Hérodias s'est révélée performative.

Jusqu'ici, nous avons considéré Hérodias comme un adjuvant potentiel d'Antipas. En tant qu'épouse, elle s'est montrée décevante; comme alliée politique elle est maladroite; mais comme magicienne elle est efficace. Il nous faut donc à présent inverser le sens de notre observation et considérer en elle-même Hérodias.

Hérodias apparut, - coiffée d'une mitre assyrienne qu'une mentonnière attachait à son front: ses cheveux en spirale s'étendaient sur un péplos d'écarlate, fendu dans la longueur des manches. Deux monstres en pierre, pareils à ceux du trésor des Atrides, se dressant con tre la porte, elle ressemblait à Cybèle accotée de ses lions (195).

Flaubert compare Hérodias à la déesse de la terre d'origine phrygienne assimilée à la déesse Astarté. Margaret Lowe ${ }^{6}$ nous précise qu'il avait noté: Hérodias est "en haut des tribunes; et elle doit être en haut car dans la religion syrienne les femmes sont toutes-puissantes et elles sont en haut". A partir de cette remarque, il devient possible de revisiter tout le conte. ${ }^{7}$ Tout d'abord, pour un homme du dix- neuvième siècle, Astarté représente la grande déesse de la luxure et c'est ainsi que Iaokanann la perçoit au chapitre II. Cybèle dompte aussi les bêtes sauvages et nous avons déjà signalé son pouvoir de mettre fin à la cohue des peuples exaltés, d'un seul mot. Cybèle est egalement une déesse-mère et Hérodias possède une enfant. Si l'on accepte l'association d'Hérodias à Cybèle 
il devient nécessaire de s'interroger sur le personnage d'Antipas. Dans les diverses légendes concernant Cybèle, la déesse se trouve associée à un dieu inférieur: Attis. Ce dernier est considéré comme un dieu de la végétation, ${ }^{8}$ or en tant que gouverneur, Antipas est responsable des productions agricoles de la région. $\mathrm{Au}$ début du récit, il détourne la vue du temple "pour contempler les palmiers de Jéricho" (140-41), déjà il avait remarqué "les grenadiers d'Esquol" (140), les "vignes de Sorek" (140) et les "champs de sésame de Karmel" (140). Par ailleurs, lorsque le culte de Cybèle se répandit à travers la Grèce, la figure d'Attis fut modifiée.' Il fut représenté comme un berger dont Cybèle serait tombée amoureuse. Or Hérodias, l'orgueilleuse patricienne, rappelle à Antipas ses origines plébéiennes et notamment que certains de ses ancêtres étaient bergers. Cybèle choisit ce berger comme prêtre et Antipas attribue à Hérodias les pouvoirs surnaturels des dieux. Notons encore que la citadelle de Machaerous est bâtie "sur un pic de basalte" (139) et une pierre noire symbolise la déesse." Flaubert compare la citadelle à "une couronne de pierres" (139), l'image d'une tour crénelée qui se dégage évoque la couronne en forme de tours qui est l'attribut des déesses-mères d'Asie. Enfin, Cybèle fut adorée sur les montagnes. En poursuivant le parallèle entre l'histoire d'Antipas et celle d'Attis, peut-être le conte révèlera-t-il une complexité nouvelle.

Selon la légende, Cybèle aurait imposé à Attis un voeu de chasteté qu'il rompit en épousant la fille de la rivière Sangarious. Sans doute pouvons-nous remarquer que Iaokanann est lié au Jourdain dans lequel il baptisa. D'ailleurs, Antipas dit de Iaokanann: "[s]a puissance est forte!.... [m]algré moi, je l'aime!" (156), ce qu'Hérodias, l'épouse humaine, ne peut tolérer. Lorsque la déesse apprit la trahison du prêtre Attis, elle le frappa d'un délire frénétique au cours duquel il se mutila. Or Antipas est pris de délire durant la danse de Salomé. En décapitant Iaokanann, c'est lui-même qu'il assassine, comme l'a fait remarquer C.H. Wake. ${ }^{11}$ En effet, son pouvoir se retourne contre sa volonté et il est contraint de céder aux forces qui le manipulent. La mutilation n'est pas du même ordre, mais Antipas a perdu la raison tout comme Attis. L'aspect naturel de la danse se trouve renforcé par le contraste entre les descriptions de Salomé avant, pendant et après son exécution. La banalité de la phrase "[u]ne jeune fille venait d'entrer" (195) ne laisse aucunement présager des débordements de sensualité et d'érotisme qui vont suivre. Après la danse, son silence, son obéissance "au claquement de doigts" (199) anonyme, son zézaiement, son "air enfantin" (199) son oubli du nom de la victime et son sourire créent un contraste violent avec le spectacle qui a précédé. Pendant la danse la déesse s'est réellement incarnée dans l'enfant. Ce n'est donc pas à sa sensualité incontrôlée qu'Antipas a cédé mais à la toute- puissance de la chair ("[c]'est Vénus tout entière à sa proie attachée"). La légende se poursuit par la métamorphose d'Attis en pin par Cybèle. A la fin du récit, Antipas semble subir une sorte de pétrification: "il ne resta plus dans la salle qu'Antipas, les mains contre les tempes, et regardant toujours la tête coupée" (202).

Le couple Hérodias-Antipas semble donc fonctionner à deux niveaux. Au niveau humain, un souverain de l'Ancien Orient finit par perdre son pouvoir en s'accrochant à de vieilles puissances dont la force se trouve abolie par de nouvelles qui les dépassent en se jouant d'elles et de leurs représentants. Au niveau des dieux, dans l'accomplissement-même du destin des anciennes déités païennes s'amorce l'avènement du dieu chrétien. S'il est vrai, comme le dit J.D. 
Hubert ${ }^{12}$ que "if we view the entire situation from the standpoint of chronology, we can sense a waning of the old order, the coming into being of the new, for the day of Salomé's danse and Iaokanann's beheading, coïnciding with the Tetrarch's birthday, stands at a threshold in time" (248-49), il ne marque peutêtre pas "the separation between the two Testaments" (ibid), mais l'émergence du monde chrétien au sein du monde païen.

Cependant, le mythe d'Attis est ausi un mythe de la résurrection, c'està-dire de la régénération. A condition de n'envisager que les aspects majeurs de chacun des cultes, le nouveau apparait comme une métamorphose de l'ancien. En effet, si dans la mort de Iaokanann s'accomplit celle d'Antipas-Attis, l'on peut aussi convenir que Iaokanann ressuscite d'une certaine façon dans le Christ, qui lui-même par sa mort régénère le monde et ressuscite à son tour. Dans cette suite de réincarnations, et de résurrections salvatrices, qui s'accordent avec le culte des Anciens et le Nouveau Testament, Flaubert me semble avoir exprimé son intérêt pour un synchrétisme religieux semblable à celui qu'il laissait deviner dans certaines de ses lettres. Car, lorsqu'il écrivit: "[l]'histoire d'Hérodias telle que je la comprends, n'a aucun rapport avec la religion," ${ }^{13}$ on peut entendre par là, comme il le dit lui-même, qu'il ne veut pas faire édifiant, une apologie chrétienne n'est pas dans ses projets. Mais il écrivit aussi: "On ne vit pas sans religion," 14 "ce qui m'attire par-dessus tout, c'est la religion. Je veux dire toutes les religions, pas plus l'une que l'autre. J'y découvre, moi, nécessité et instinct." 15 Ainsi peut-on extrapoler qu'il s'intéresse au fond commun de toutes les religions, à ce qui fascine universellement l'homme.

Jusqu'ici, nous avons essayé de montrer que deux trames se tissent en Hérodias. La première nous raconte comment le Tétrarque Antipas perdit son pouvoir sous l'influence d'une femme pernicieuse et retorse: Hérodias. Le second reprend le mythe du couple divin Attis-Cybèle et là encore c'est sous l'influence de Cybèle-Hérodias que s'accomplit la régénération du monde. Mais il ne me semble pas pouvoir conclure ici sur la nécessité du titre Hérodias car au fond, de quelque façon qu'on l'envisage c'est bien Iaokanann qui en mourant triomphe. En effet, dans tous les cas, il remplit sa mission et accomplit son destin: pour que le Christ "croisse, il faut qu['il] diminue" (145), en mourant il fait advenir le règne de la Bonne Nouvelle. La raison pour laquelle son nom ne fut pas associé au titre demeure un mystère qu'il nous faut tâcher d'éclaircir.

La complexité du personnage de Iaokanann me semble provenir du fait qu'il n'est pas un personnage de roman tel qu'on l'entend traditionnellement. Autant Flaubert nous permet de comprendre la psychologie d'Hérode et celle d'Hérodias, autant nous ne possédons aucun indice pour analyser celle de Iaokanann. En fait, Iaokanann constitue la cristallisation des forces surnaturelles ou tout simplement de l'énergie qui permet la régénération de la terre. Nous prenons l'habitude de l'appeler Iaokanann, mais Flaubert ne le nomme pas tout d'abord. "Tout à coup, une voix lointaine, comme échappée des profondeurs de la terre, fit pâlir le Tétrarque" (142). Il surprend, il constitue une menace latente, pourtant il demeure une voix sans visage, impossible à emprisonner, irréductible. En l'entendant on pense aux grondements qui précèdent les catastrophes naturelles, éruptions volcaniques, tremblements de terre ou orages. S'il n'y avait le mot "voix", l'on ne songerait pas à un homme. C'est un mouvement de fond encore lointain, une armée en marche, mais pas un personnage comme Antipas ou Mannaeï. D'ailleurs, si le narrateur l'appelle "Iaokanann", ces deux derniers 
ne semblent oser le nommer: "Où est-il?" (143) demande le premier, "Là! Toujours!" (143) répond le bourreau. Iaokanann est associé à l'ombre ("profondeurs de la terre" (142), "dans les ténèbres (145), "le soir") et au mystère ("Ils ont échangé avec lui des paroles mystérieuses" (143)) à la fois celui qu'il porte et celui où on le tient, à l'inconnaissable enfin.

Dans tout le premier chapitre, les paroles de Iaokanann sont rapportées par les autres personnages sans que l'on assiste à leur première profération. D'abord Mannaeï dit: “je le vois qui marche dans les ténèbres, en répétant: 'Qu'importe? Pour qu'Il grandisse, il faut que je diminue!' “ (143). Puis Hérodias évoque pour Antipas les injures que Iaokanann lui a lancées, mais elle ne reproduit pas fidèlement ses propos, qui d'ailleurs ne lui appartiennent pas en propre puisqu'ils contiennent toutes les malédictions des prophètes. Cette présentation renforce notre impression que cette voix n'est pas celle d'un homme, mais que tout en émanant d'abord d'une certaine bouche humaine, elle est en fait désincarnée. Iaokanann n'est que le premier relais transmetteur de paroles qui le dépassent et se répandent au-delà de lui, sans son contrôle.

Au chapitre II, l'on entend et l'on voit enfin Iaokanann, dont l'existence semblait mythique. De "sa voix caverneuse" (171) - qui reprend l'expression "échappée des profondeurs de la terre" (142) —, il confirme qu'il ne menace pas en son nom mais en celui de "l'Eternel" (172). Mais son discours demeure mystérieux pour son audience: "De quel conquérant parlait-il?" (172). De fait, même ses promesses douces se transforment en menaces pour Antipas, "l'existence d'un fils de David l'outrageant comme une menace" (174). Le mystère de Iaokanann ne provenait donc pas uniquement de sa réclusion, il constitue son trait dominant. Seules ses menaces contre Hérodias sont explicites et elle les comprend. Au reste, il la condamne sans rémission possible, ne lui offrant aucune chance de repentir. Peut-être dans cette dichotomie qui s'établit ici entre Hérodias et les autres peut-on voir la confirmation qu'au coeur de ce conte se situe le combat entre Cybèle, déesse de la terre en son état primitif et ce dieu que Iaokanann nomme 'l'Eternel". En effet, si aucun' pardon ne peut être accordé à Cybèle-Hérodias, c'est qu'elle doit disparaître pour que la terre entame un nouveau cycle.

De ce moment, l'on n'entendra plus Iaokanann, mais son mystère grandira jusqu'à sa mort. Ainsi Mannaeï, le mystique samaritain, faillira au moment de l'exécuter, il aura des hallucinations: "Il avait aperçu devant la fosse le Grand Ange des Samaritains, tout couvert d'yeux et brandissant un immense glaive, rouge, et dentelé comme une flamme" (200). Ici Flaubert me semble renforcer sa vision religieuse synchrétique, en introduisant dans le jeu des forces qui amènent une nouvelle ère cet "Ange des Samaritains" opposé aux Juifs, qui semble protéger Iaokanann et jette Mannaeï dans la confusion, mais en réalité diffère l'accomplissement de la mission du prophète et donc l'avènement du nouveau monde.

Au-delà de la mort, Iaokanann paraît encore posséder une volonté, car l'expression "[l]a tête entra" (201) nous donne le sentiment qu'elle se meut ellemême. En l'examinant, les capitaines romains nous permettent de constater combien la blessure infligée au prophète visait surtout à le faire taire. En effet, le cou qui aurait dû être tranché n'est pas mentionné, mais Mannaeï a "entamé la machoire. Une convulsion tirait les coins de la bouche. Du sang caillé déjà parsemait la barbe" (202); l'attaque s'est concentrée autour de la bouche. Or, en cette 
tactique se situe l'erreur des défenseurs de l'ancien ordre, car la parole de Iaokanann ne siège déjà plus en lui, elle a été propagée par d'autres hommes et Jésus peut désormais en compléter le message. Cependant, le mystère ne s'éclaircit que pour Phanuel grâce à la réponse apportée par les messagers. La lourdeur de la tête durant son transport représente à la fois le poids de l'espoir et la garantie qu'il est fondé. Ainsi se confirme que Iaokanann n'est pas le représentant d'un être humain, mais le véhicule provisoire d'une voix céleste, la matérialisation de la mutation en train de s'accomplir. Pourtant, le narrateur utilise l'expression "être humain" à propos de Iaokanann, le mystère qui l'entoure ne suffirait-il pas à lui refuser la qualité de personnage?

En fait, dans le premier et le deuxième chapitre, Iaokanann est explicitement comparé à un animal. "[I]l a l'air tranquille d'une bête malade" (145), dit Mannaeï tandis qu'Hérodias le décrit ainsi: "Il avait une peau de chameau autour des reins, et sa tête ressemblait à celle d'un lion. .... Ses prunelles flamboyaient; sa voix rugissait" (150). Plus tard, le narrateur - juste après avoir appelé Iaokanann "[u]n être humain" (170) et comme pour le nier - lui attribue "de longs cheveux se confondant avec les poils de bête qui garnissaient son dos" (170) et assimile sa prison à "son antre" (170). Iaokanann lui-même dit: "Je crierai comme un ours, comme un âne sauvage" (174). Or on se souvient que Cybèle-Hérodias domptait les animaux sauvages. Ici Iaokanann fonctionne comme un symbole de sa réduction à l'impuissance, car contre cette bête, féroce à son égard, elle ne peut rien, il demeure irréductible. D'ailleurs, il est clair dès le premier chapitre que Iaokanann est indestructible: "Quand on l'avait pris et lié avec des cordes, les soldats devaient le poignarder s'il résistait; il s'était montré doux. On avait mis des serpents dans sa prison; ils étaient morts" (151). L'erreur d'Antipas et d'Hérodias est justement qu'ils s'obstinent à prendre Iaokanann pour un "homme" (150), un "prisonnier" (177), dont la "puissance est forte" (156). En réalité, il n'est qu'énergie concentrée, évocation d'une force souterraine qui se répand: "Il a même dépêché de Machaerous des hommes qui bouleversent [les] provinces" d'Antipas, "[I]l ira chez les Arabes, les Gaulois, les Scythes. Son oeuvre doit s'étendre jusqu'au bout de la terre!" (156). "Son nom circulait" (171), "[l]a voix grossissait, se développait, roulait avec des déchirements de tonnerre, et, l'écho dans la montagne la répétant, elle foudroyait Machaerous d'éclats multipliés" (176).

Ainsi se confirme la nature inhumaine de Iaokanann, vecteur de l'énergie nouvelle, tonnerre d'un nouveau Zeus. D'ailleurs, lorsque "renversa[nt] la tête" (175), son visage a "l'air d'une broussaille, où étincelaient deux charbons" (175), il prend en quelque sorte feu se révélant pure énergie. Lisant "Les paupières closes étaient blêmes comme des coquilles; et les candélabres à l'entour envoyaient des rayons" (202), hommes du vingtième siècle, nous songeons que de la fantastique énergie qui vient d'être relachée, comme dans une explosion nucléaire, il reste encore la trace radioactive. Tout s'est accompli, mais la tête de Iaokanann comme dans les tableaux de Gustave Moreau irradie encore. Parallèlement, un double renversement s'est effectué qui affirme la réalisation de la conversion du monde.

Tout au long des deux premiers chapitres, nous avons déjà noté que Iaokanann est associé aux ténèbres. Au contraire, Antipas est associé à la lumière: nous le découvrons à l'aube, alors que le soleil rougit Machaerous; lorsqu'il parle de Iaokanann pour la première fois avec Phanuel, il est couché 
sous "un bouclier d'or" (155) qui luit "comme un soleil" (155); et tandis que Iaokanann "dispara[ît] dans les profondeurs de son antre" (170), "le soleil faisait briller la pointe des tiares, le pommeau des glaives" (170). Déjà Iaokanann prédit la disparition de la lumière pour les peuples fidèles à l'ordre ancien, à travers ses mystérieuses métaphores: "comme l'avorton d'une femme qui ne voit pas le soleil" (172), "[i]l faudra.... te réfugier dans les cyprès...., dans les cavernes" (172), "[o]n ira, la nuit, chercher son pain" (172), enfin "les chacals" évoquent des ténèbres généralisées. Or, dans la scène finale, alors que la tête coupée rayonne dans son nimbe, "les flambeaux s'éteignaient" (202) et Antipas demeure dans le noir. Hérode-Attis, serviteur des anciens dieux qui possédait le pouvoir d'enfermer Iaokanann se retrouve à son tour privé de lumière. De même Iaokanann, longtemps assimilé à une "bête furieuse" (156) enflammée, dévastatrice, hurlante et rouge, après sa mort prend une transparence empreinte de paix, irradie une lumière froide: "Les paupières closes étaient blêmes comme des coquilles" (202). Au contraire, Hérodias, qui était apparue pour la fête derrière les panneaux de "la tribune d'or" (194), "à la splendeur des cierges" (195), en pleine gloire, presque irréelle dans sa beauté divine et sereine, se transforme en animal sauvage, enragé et exempt de dignité lorsque Mannaeï échoue: "La fureur d'Hérodias dégorgea en un torrent d'injures populacières et sanglantes. Elle se cassa les ongles au grillage de la tribune, et les deux lions sculptés semblaient mordre ses épaules et rugir comme elle" (200). Iaokanann a donc bien inversé leurs rôles et fait tomber Cybèle- Hérodias de son piédestal en la rabaissant au niveau des bêtes sauvages dont elle était la maîtresse.

Le titre du conte semble à présent s'éclairer. En effet, au niveau humain, Hérodias manoeuvre tout au long du conte Antipas. Elle possède en réalité le pouvoir qu'il semble détenir puisque c'est sur elle et ses envoûtements qu'il le fait reposer. Au niveau du mythe, elle contrôle la vie du dieu inférieur AntipasAttis. Certes, Cybèle-Hérodias se perd elle-même par ses propres machinations, mais peut-on dire qu'elle est vaincue par Iaokanann? Ce dernier ne me semble pas constituer un personnage, il n'est qu'un représentant, le point sur lequel se cristallise la lutte des dieux. A la fois 'innocent massacré' et 'plaie d'Egypte,' il est le véhicule d'une nouvelle naissance, mais il n'est pas un personnage auquel opposer les deux autres. Alors, celui qui devrait donner son nom au récit n'est-il pas le Dieu de la Bonne Nouvelle? Mais Flaubert se moque bien de constituer un témoignage chrétien. D'ailleurs, si le Christ sauve les hommes par sa mort et sa résurrection, le culte d'Attis comporte également la mort et la résurrection du dieu pour assurer la régénération du monde. Peut-être alors Flaubert en nommant son conte Hérodias voulait-il nous faire remarquer combien le nouveau n'est qu'une métamorphose du même. Aussi éloignés qu'ils semblent l'un de l'autre, Antipas-Attis et Iaokanann-Christ peuvent être envisagés comme deux versions du même mythe et celle qui permet la résurrection de l'un en l'autre, Hérodias, femme, déesse, jeune fille et finalement "vieille femme" (201), mérite bien le titre du conte. S'il est vrai que ce qui distingue un conte d'une nouvelle est sa conclusion morale, la tolérance est peut-être le message le plus caché du texte. D'ailleurs Flaubert ne dit-il pas lui-même: “Comprenons chaque chose et n'en blâmons aucune. C'est le moyen de savoir beaucoup et d'être calme." 


\section{Notes}

${ }^{1}$ Toutes les citations, référées (n), sont extraites de Gustave Flaubert,Trois Contes, ed. Edouard Maynial (Paris: Garnier, 1969).

${ }^{2}$ C.H. Wake, "Symbolism in Flaubert's Hérodias: An Interpretation," Forum for Modern Languages Studies 4 (1968): 322-329.

${ }^{3}$ Jane Robertson, "The Structure of Hérodias, “ French Studies 36 (Apr. 1982): 171-182. p. 172.

${ }^{4}$ Flaubert écrivit en effet dans une lettre datée du 17 Août 1876 publiée dans la Correspondance, vol. 7 (Paris: Conard, 1926-1933) 341, et citée par Maurice Bardèche, L'oeuvre de Flaubert: D'après ses carnets, ses études des scénarios, sa correspondance inédite, (Paris: Les Sept Couleurs, 1974) 348: "Je vois, nettement, comme je vois la Seine, la surface de la Mer Morte scintiller au soleil. Hérode et sa femme sont sur un balcon d'où l'on découvre les tuiles dorées du temple".

${ }^{5}$ C.H. Wake, 327-328.

'Margaret Lowe, "Rendre plastique", Modern Language Review, 78 (July 1983): 551-58. p. 553.

' Margaret Lowe a remarqué nombre d'indices utilisés ici dans son article "Rendre plastique" mais en en donnant, me semble-t-il, une interprétation toutà-fait différente. Elle considère en particulier que Flaubert voyait en son propre siècle la récurrence d'un culte du principe féminin.

${ }^{8}$ Dans A Dictionary of Non-Christian Religions (Philadelphia: Westminster Press, 1973) 68, Geoffrey Parrinder écrit : "The great mother-goddess of ancient Asia Minor and worshipped with the god of vegetation, her young lover Attis".

${ }^{9}$ Ces remarques concernant l'évolution des figures d'Attis et de Cybèle sont tirées de New Larousse Encyclopedia of Mythology (Felyham: Astronaut House, 1968) 150:

As the cult of Cybele spread through Greece the figure of Attis became modified. He was presented as a young and handsome shep herd from Celaenae with whom Cybèle fell in love. She chose him as her priest and imposed upon him a vow of chastity. When Attis broke his vow and espoused the daughter of the river Sangarius, Cybèle struck him with frenzied delirium in the course of which he mutilated him self. When he recovered from his madness he was on the point of killing himself when Cybele changed him into a fir tree."

${ }^{10}$ A Rome, une "black meteoric stone .... was supposed to be the throne of the goddess." (Ibid, 220)

"C. H. Wake, 323-324.

${ }^{12}$ J.D. Hubert, "Representations of Decapitation: Mallarmé's Hérodiade and Flaubert's Hérodias," French Forum 7 (1982): 245-51.

${ }^{13}$ Il s'agit d'un extrait d'une lettre adressée à Madame Régnier le 24 décembre 1876, publiée dans la Correspondance, vol. 7, 376 et citée dans Joyce H. Cannon, "Flaubert's Documentation for Hérodias," French Studies 14 (1960): 325-339.

${ }^{14}$ Cité dans Margaret G. Tillet, "An Approach to Hérodias," French Studies 21 (1967): 24-31. p. 26.

${ }^{15}$ Cité dans Margaret G. Tillet, 25. 


\section{Ouvrages cités}

Bardèche, Maurice. L'Oeuvre de Flaubert: D'après ses carnets, ses études des scénarios, sa correspondance inédite. Paris: Les Sept Couleurs, 1974.

Beck, William J. "Flaubert's Tripartite Concept of History and Trois Contes." College Language Association Journal 21 (1978) : 7478.

Bertrand, Marc. "Paroles et silences dans les Trois Contes de Flaubert." Stanford French Review 1 (1977) : 191-203.

Bonaccorso, Giovanni. "L'influence de l'Orient dans lesTrois Contes." Les Amis de Flaubert Mai 1977 : 9-21.

--.. "L'influence de l'Orient dans les Trois Contes." Les Amis de Flaubert Déc. 1977 : 29-41.

Brée, Germaine. "Flaubert et la critique récente." Essais sur Flaubert : En

l'honeur du professeur Don Demorest. Paris : Nizet, 1979. 31-38. Burns, C.A. "The Manuscripts of Flaubert's Trois Contes." French Studies 8 (1954): 4.

Cancalon, Elaine D. "La symbolique de l'espace dans Hérodias." South Atlantic Bulletin 40, i (1975) : 23-28.

Cannon, Joyce H. "Flaubert's Documentation for Hérodias." French Studies 14 (1960) : 325-339.

--.. "Flaubert's Search for Form in Hérodias." Modern Language Review 57 (1962) : 195-203.

Carlut, Charles, Pierre H. Dube and Jean-Raymond Dugan. A Concordance to Flaubert's Trois Contes. New-York : Garland Reference Library of the Humanities, 1979.

Combescot, Pierre. "Le mirage de l'Orient."Magazine Littéraire Jan. $1976: 18-19$.

Czyba, Lucette. Mythes et idéologies de la fenme dans les romans de Flaubert. Lyon: P.U. de Lyon, 1983.

Danger, Pierre. Sensations et objets dans les romans de Flaubert. Paris :

Colin, 1973.

Debray-Genette, Raymonde. "Du mode narratif dans les Trois Contes."

Littérature 2 (1971) : 39-70.

---. "Re-présentation d'Hérodias." Production du sens chez Flaubert.

Paris : Union Générale d'Editions, 1975. 328-344.

Debray-Genette, Raymonde, and Susan Houston. "Profane, Sacred :

Disorder of Utterance in Trois Contes." Flaubert and

Postmodernism. Ed. Naomi Schor and Henry F. Majewski. Lincoln :

University of Nebraska Press, 1984. 13-19.

Donato, Eugenio. "Flaubert and the Question of History : Notes for a

Critical Anthology." Modern Language Notes 91 (1976) : 850-870.

Duncan, Phillip A. "The Equation of Theme and Spatial Form in Flaubert's

Hérodias." Studies in Short Fiction 14 (1977) : 129-136. 
Flaubert, Gustave. Trois Contes. Ed. Edouard Maynial. Paris : Garnier, 1969.

Fletcher, John. A Critical Commentary on Flaubert's Trois Contes. London : Macmillan, 1968.

Genette, Gérard. "Demotivation in Hérodias." Flaubert and

Postmodernism. Trans. Marlena Corcoran. Ed. Naomi Schor and Henry F. Majewski. Lincoln : University of Nebraska Press, 1984.

Gothot-Mersch, Claudine. "Sur le narrateur chez Flaubert."Nineteenth

Century French Studies. Spring 1984 : 344-365.

Hanoulle, Marie-Julie. "Quelques manifestations du discours dansTrois

Contes."Poétique 9 (1972) : 41-49.

Hubert, J.D. "Representations of Decapitation : Mallarmé's Hérodiade and Flaubert's Hérodias." French Forum (1982) : 245-251.

Issacharoff, Michael. L'Espace et la nouvelle : Flaubert, Huysmans,

Ionesco, Sartre, Camus. Paris : Corti, 1976.

---. "Hérodias et la symbolique combinatoire des Trois Contes."

Languages de Flaubert. Paris : Lettres Modernes Minard, 1976.

--. "Trois Contes et le problème de la non-linéarité." Littérature 15

(1974) : 27-40.

Leal, R.B. "Spatiality and Structure in Flaubert's Hérodias." Modern Language Review Oct. 1985 : 810-816.

Leening, David A. Mythology : the Voyage of the Hero. Philadelphia : Lippincott, 1973.

Lowe, Margaret. "Hérodias, the Second Empire and La Tête d'Orphée." French Studies Bulletin Summer 1982 : 6-8.

---. "Hérodias, roman du second Empire."French Studies Bulletin Winter 1981-82:9-10.

--.. "Rendre plastique : Flaubert's treatment of the Female Principle in Hérodias." Modern Language Review July 1983 : 551-558.

Malinowski, Wieslaw. "Hérodias de Flaubert et Thaïs de France. Quelques affinités artistiques et intellectuelles." Studia Romanica Posnaniensia 7 (1981) : 29-38.

Marotin, François. "Les Trois Contes : un carrefour dans l'oeuvre de

Flaubert." Frontière du Conte. Ed. François Marotin. Paris : CNRS, 1982. 111-118.

Maynial, Edouard. Introd. Trois Contes : Un coeur simple : La légende de Saint-Julien l'Hospitalier : Hérodias. By Flaubert, Gustave. Paris : Garnier, 1960.

Neefs, Jacques. "Le récit de l'édifice des croyances : Trois Contes." Flaubert : la dimension du texte. Ed. P.M. Wetherhill. Manchester : Manchester P.U., 1982. 121-140.

New Larousse Encyclopedia of Mythology. Felyham : Astronaut House, 1968.

Nykrog, Per. "Les Trois Contes dans l'évolution de la structure thématique chez Flaubert." Romantisme 6 (1973) : 55-66. 
O'Connor, John R. "Flaubert's Trois Contes and the Figure of the Double

Cone."PMLA 95 (1980) : 812-826.

Parrinder, Geoffrey. A Dictionary of Non-Christian Religions.

Philadelphia : The Westminster Press, 1971.

Peterson, Carla L. "The Trinity in Flaubert's Trois Contes : Deconstructing

History." French Forum Sep. 1983 : 243-258.

Raitt, Allan W. "Etat présent des études sur Flaubert." L'Information

Littéraire 35 (1983) : 18-25.

---. "Flaubert and the Art of the Short Story."Essays by Divers Hands 38

(1975) : 112-126.

Riesch, Joseph C. "Those who see God in Flaubert'sTrois Contes."

Renascence Summer 1984 : 219-229.

Robert, Marthe.En haine du roman : étude sur Flaubert. Paris : Balland, 1982.

Robertson, Jane. "The Structure of Hérodias."French Studies Apr.

1982: 171-182.

Sachs, Murray. "Flaubert's Trois Contes : The Reconquest of art." L'Esprit Créateur 10 (1970) : 62-74.

Selvin, Susan C. "Spatial Form in Flaubert's Trois Contes."Romanic

Review Mar. 1983 : 202-220.

Shapira, Charlotte. "La Relative introduite dans et par les Trois Contes."

Romance Notes Fall 1983 : 22-26.

Shepler, Frederic J. "La mort et la rédemption dans lesTrois Contes de

Flaubert." Neophilologus 56(1972) : 407-416.

Thompson, Patrice : "La méthode de composition de Flaubert dans

Hérodias." Actes de la Journée Flaubert. Fribourg : Ed.

Universitaires, 1981. 49-63.

Tillet, Margaret G. "An Approach to Hérodias." French Studies 21 (1967):

24-31.

Tondeur, Lise-Claire. "Flaubert et Sade, ou la fascination de l'excès."

Nineteenth Century French Studies Fall-Winter 1981-82 : 75-84.

Tournier, Michel. "La dimension mythologique." NRF Oct. 1972 : 124-29.

--. "Nécessité et liberté dans les Trois Contes."Le vol du vampire. Paris :

Mercure de France, 1981. 161-168.

Unwin, Thimoty A. "La présence de Flaubert dans les Trois Contes."Les

Amis de Flaubert Déc. 1978 : 12-20.

Vigée, Claude. "L'ambivalence de l'image mythique chez Flaubert."Essais sur Flaubert : En l'honneur du professeur Don Demorest. Ed.

Charles Carnut. Paris : Nizet, 1979. 155-183.

Wake, C.H. "Symbolism in Flaubert's Hérodias : an Interpretation." Forum for Modern Languages Studies 4 (1968) : 322-329.

Zagora, Helen G. "A Historical Approach of Flaubert's Hérodias." The Legend of Salomé. Genève : Droz, 1960. 
